\title{
Determinants of Birth Asphyxia Among Newborns in Debre Berhan Referral Hospital, Debre Berhan, Ethiopia: a Case-control Study
}

Sisay Shine Tegegnework ( $\nabla$ sisayask@gmail.com )

Debre Berhan University

\section{Yeshfanos Tekola Gebre}

Debre Berhan University

Sindew Mahmud Ahmed

Kotebe Metropolitan University

\section{Abrham Shitaw Tewachew}

Debre Berhan University

\section{Research Article}

Keywords: Birth asphyxia, Determinant, Newborn, Debre Berhan, Ethiopia

Posted Date: September 8th, 2021

DOI: https://doi.org/10.21203/rs.3.rs-870893/v1

License: (c) (i) This work is licensed under a Creative Commons Attribution 4.0 International License. Read Full License 


\section{Abstract}

Background: Birth asphyxia is the major public health problem in the world. It is estimated that around $23 \%$ of all newborn deaths are caused by birth asphyxia worldwide. Birth asphyxia is the top three causes of newborn deaths in sub-Saharan Africa and more than one-third of deaths in Ethiopia. Therefore, the aim of this study was to identify determinants of birth asphyxia which can play a crucial role to decrease the death of newborns.

Methods: Unmatched case-control study design was implemented among 276 (92 cases and 184 controls) newborns from January 1 st to March 30th, 2020. A systematic sampling technique was used to select the study participants. Data were collected by using a semi-structured interviewer-administered questionnaire and document review by trained nurses and midwives who work at the delivery ward of the hospitals. Bivariate logistic regression analysis was done to identify determinants of birth asphyxia. Adjusted odds ratios with $95 \%$ confidence intervals and p-value less than 0.05 were used to assess the level of significance.

Results: In this study, maternal education of being can't read \& write [AOR $=4.7,95 \% \mathrm{Cl}:(1.2,11.9)]$, antepartum hemorrhage $[A O R=7.7,95 \% \mathrm{Cl}:(1.5,18.5)]$, prolonged labor $[\mathrm{AOR}=13.5,95 \% \mathrm{Cl}:(2.0,19.4)]$, meconium stained amniotic fluid [AOR $=11.3,95 \% \mathrm{Cl}:(2.7,39.5)]$, breech fetal presentation [AOR $=4.5$, $95 \% \mathrm{Cl}:(2.0,8.4)]$ and preterm birth [AOR: $4.1,95 \% \mathrm{Cl}:(1.8,9.2)]$ were factors which showed significantly associated with birth asphyxia among newborns.

Conclusions: In this study, maternal education can't read \& write, antepartum hemorrhage, prolonged labour, stained amniotic fluid, breech fetal presentation, preterm birth were significantly associated with birth asphyxia. So, educating mothers to have continuous follow-up during the pregnancy period and provide quality care to the women in labour through close monitoring of the fetus presentation were recommended to reduce birth asphyxia.

\section{Introduction}

Birth asphyxia is defined as the failure of the newborn to initiate and sustain adequate respiration after delivery [1]. It is existence of three factors like metabolic or mixed academia $(\mathrm{Ph}<7)$ which is determined by umbilical cord arterial blood samples; APGAR score of $<3$ for longer than 5 minute; neurological manifestations and multisystem organ dysfunction [2]. It is the major public health problem among under-five children in the world. Globally, 4 million or $23.0 \%$ of newborn deaths occur yearly due to birth asphyxia, representing the fifth (38.0\%) largest cause of under-5 year's children deaths [3]. The problem is severe in developing countries at which 120 million newborns develop birth asphyxia and the cause for 900,000 deaths every year. In sub-Saharan African countries $24.0 \%$ of newborn deaths were due to birth asphyxia [4].

In Ethiopia, birth asphyxia is the cause of more than one-third of newborn deaths [5]. The effect of birth asphyxia is not limited only to death but also has a short and long-term neurodevelopment squeal 
including cognitive and motor disabilities which are almost untreatable [6, 7]. In order to alleviate this problem, national newborn training and guidelines are well established for those health professionals who were attending labor at any health facility level. There is also a child survival strategy in Ethiopia that connects essential maternal, newborn, and child health packages throughout adolescence, pregnancy, childbirth, postnatal and newborn periods, and into childhood building upon their natural interactions throughout life. Even if such strategies were applied by Ethiopian health policy the problem of newborn death was still high in the country $[5,8]$.

Many studies in different parts of Ethiopia showed that parity [9], anemia [10, 11], antepartum hemorrhage [12], prolonged labor [9], preterm birth [12], meconium-stained liquor [12, 13], pregnancyinduced hypertension [10], and mode of delivery [14] were significant predictors of birth asphyxia. However, information related to determinants of newborn asphyxia in Debre Berhan referral hospitals is limited. Therefore, the aim of this study was to identify determinants of birth asphyxia among newborns in Debre Berhan Referral Hospital, Debre Behan, Ethiopia. The findings will help to improve health care providers' and women's knowledge on birth asphyxia during labor. In addition, the information will also give a guide for policymakers and stakeholders with updated information for future planning and interventions.

\section{Methods}

Study area and period: The study was conducted in Debre Berhan referral hospital at Debre Berhan town. It is located in North Shoa Zone, Amara regional state, North East part of Ethiopia. It is $130 \mathrm{~km}$ far from the capital city Addis Ababa, and $695 \mathrm{~km}$ far from the regional city Bahir Dar. It is one of the largest public referral hospitals in the zone, providing preventive, curative and rehabilitative services to about more 3 million people in the catchment area. It also provides delivery service 24 hours a day, 7 days a week by 24 midwives, 3 general practitioners and 4 gynecologists who assist about 3366 deliveries annually. In NICU ward there are 22 nurses, 3 general practitioners and 2 pediatricians. The total admission of NICU wards from 2019 report 1056 neonates 313 by birth asphyxia. The study was conducted from January 1st to March 30th, 2020.

Study design: An institution-based unmatched case-control study design was employed to assess determinants of birth asphyxia among newborns in Debre Berhan Referral Hospital.

Population: All newborns delivered in Debre Berhan referral hospital were the source population and all newborns delivered in the hospital during the study period were the study population. Newborns who had heart deformity and more than one malformation were excluded from the study.

Sample size determination: The sample size was determined by Epi Info 7 version software package for the unmatched case control study. By considering, 29.4\% proportion of controls exposed and odds ratio of 2 for low gestational age from a previous study conducted in Ethiopia Tigray region [12], with an assumption of, $95 \%$ confidence interval, $80 \%$ power of the study, two- to- one control to cases ratio, and $5 \%$ non-response rate. The final sample size was 276 ( 92 cases and 184 controls). 
Sampling procedure: A systematic random sampling technique was used to select the study participants. Every third study subjects were included for both cases and controls. On the first day of data collection the first study participant was taken by using simple random sampling technique from the registration book.

Data collection tools and procedure: The data were collected by using interviewer administered structured questionnaire adapted from different literatures [7, 12], and document review. The questionnaire was first prepared in English and translated to local language Amhric and back translates to English to keep its consistency. The questionnaire was pre-tested in another health facilities and essential amendment were made based on the results. Data were collected by four midwives and two supervisors who have en experience of work on neonatal intensive care unit of the hospital.

\section{Operational definition:}

Cases: When the newborn has at least one of the following signs, not breathing, gasping, $<30$ breaths per minute or $<7$ APGAR score [15].

Outcome variable: Birth asphyxia

\section{Independent variable}

I. Socio demographic determinants: age, marital status, residence, educational status, maternal occupation

II. Ante partum determinants: parity, pregnancy induced hypertension, anemia, ante-partum hemorrhage, ANC follow up, chronic disease

III. Intra-partum determinants: fetal presentation, duration of labor, amniotic fluid, type of labor, mode of delivery, labor attendant, PROM

IV. Neonatal determinants: Sex, birth weight, gestational age

Data quality control: The quality of data was assured by using properly designed and validated questionnaire by expertise. Training was given for the data collectors and supervisors on how to collect the data for three days. Pre-testing was performed in another health facility and essential amendment was made on the questionnaire. The principal investigator and supervisors were made a day to day on site supervision and check the collected data for completeness, clarity and consistency.

Data processing and analysis: The data was coded and entered to Epi-data version 3.1 and exported to SPSS Version 21 for data analysis. Descriptive statistics were performed and presented in the form of means, standard deviations, frequencies and percentages. Binary logistic regression was computed to identify determinates of birth asphyxia. Variable which have $p$-value less than 0.25 in binary logistic regression analysis were transferred to multiple logistic regression model to see the relative effect of the confounder. Model goodness of fit test was checked by using Hosmer Lemeshow goodness of fit and Multicolinearity was assessed by variance inflation factor (VIF) among variables. Adjusted odd ratios 
with $95 \%$ confidence interval and P-value less than 0.05 was used to identify determinates of birth asphyxia.

\section{Results}

\section{Socio-demographic characteristics of the study participants}

A total of 276 (92 cases and 184 controls) were participated in the study with a response rate of $100 \%$. About $52.2 \%$ of cases and $42.4 \%$ controls were live in rural resident and $42.4 \%$ of the mother of the cases and $6.0 \%$ controls were can't read and write in their educational status. More mother of cases $(48.9 \%)$ than controls (19.0\%) were house wife by their occupational status. The majority of mother of cases $(80.4 \%)$ and controls (95.1\%) were married in their marital status (Table 1). 
Table 1

Socio demographic characteristics of the study participants in Debre Berhan referral hospital, Debre Berhan, Ethiopia, 2020

\begin{tabular}{|c|c|c|c|}
\hline Variable & Cases (\%) & Controls (\%) & Total (\%) \\
\hline \multicolumn{4}{|l|}{ Age of the mother (year) } \\
\hline$<=19$ & $8(8.7)$ & $5(2.7)$ & $13(4.8)$ \\
\hline $20-24$ & $30(32.6)$ & $38(20.7)$ & $68(24.6)$ \\
\hline $25-29$ & $28(30.4)$ & $74(40.3)$ & $102(36.9)$ \\
\hline $30-34$ & $14(15.2)$ & $33(17.9)$ & $47(17)$ \\
\hline$>=35$ & $12(13.1)$ & $34(18.5)$ & $46(16.7)$ \\
\hline \multicolumn{4}{|l|}{ Residence } \\
\hline Urban & $44(47.8)$ & $106(57.6)$ & $150(54.3)$ \\
\hline Rural & $48(52.2)$ & $78(42.4)$ & $126(45.7)$ \\
\hline \multicolumn{4}{|l|}{ Marital status } \\
\hline Married & $74(80.4)$ & $175(95.1)$ & $249(90.2)$ \\
\hline Single & $18(19.6)$ & $9(4.9)$ & $27(9.8)$ \\
\hline \multicolumn{4}{|l|}{ Maternal education } \\
\hline Can't read and write & $39(42.4)$ & $11(6.0)$ & $50(18.1)$ \\
\hline Primary school & $22(23.9)$ & $27(14.7)$ & $49(17.8)$ \\
\hline Secondary school & $13(14.1)$ & $71(38.6)$ & $84(30.4)$ \\
\hline College/university & $18(19.6)$ & $75(40.7)$ & $93(33.7)$ \\
\hline \multicolumn{4}{|l|}{ Maternal occupation } \\
\hline House wife & $45(48.9)$ & $35(19.0)$ & $80(29.0)$ \\
\hline Merchant & $13(14.1)$ & $16(8.7)$ & $29(10.5)$ \\
\hline Farmer & $8(8.7)$ & $11(6.0)$ & $19(6.9)$ \\
\hline Private employee & $11(12.0)$ & $69(37.5)$ & $80(29.0)$ \\
\hline Government employee & 15 (16.3) & $53(28.8)$ & $68(24.6)$ \\
\hline
\end{tabular}


From the total study participants, 46 (55.4\%) mothers of the cases and $37(44.6 \%)$ mothers of the controls were premipara, 39 (61.9\%) mothers of the cases and 24 (38.1\%) mothers of the controls were less than or equals to two ANC visits, $25(78.1 \%)$ of the mothers of the cases and $7(21.9 \%)$ mothers of the controls were APH during pregnancy, $21(77.8 \%)$ mothers of the cases and $6(22.2 \%)$ of the controls had anemia and $19(73.1 \%)$ mothers of the cases and 7 (26.9\%) of the controls were PIH during pregnancy (Table 2). 
Table 2

Ante partum characteristics of the study participants in Debre Berhan referral hospital, Debre Berhan, Ethiopia, 2020

\begin{tabular}{|c|c|c|c|}
\hline Variable & $\begin{array}{l}\text { Cases } \\
(\%)\end{array}$ & $\begin{array}{l}\text { Controls } \\
\text { (\%) }\end{array}$ & Total (\%) \\
\hline \multicolumn{4}{|l|}{ Parity } \\
\hline $\begin{array}{l}1 \text { (premipara) } \\
2-4(\text { multipara }) \\
>=5 \text { (grand multipara) }\end{array}$ & $\begin{array}{l}29 \\
(31.5) \\
17 \\
(18.5)\end{array}$ & $\begin{array}{l}37(20.1) \\
129(70.1) \\
18(9.8)\end{array}$ & $\begin{array}{l}83(30.1) \\
158(57.2)\end{array}$ \\
\hline \multicolumn{4}{|l|}{ ANC follow up } \\
\hline $\begin{array}{l}<=\text { two } \\
\text { Three } \\
\text { Four and above }\end{array}$ & $\begin{array}{l}39 \\
(42.3) \\
20 \\
(21.7) \\
33 \\
(36.0)\end{array}$ & $\begin{array}{l}24(13.0) \\
19(10.3)\end{array}$ & $\begin{array}{l}63(22.8) \\
39(14.1)\end{array}$ \\
\hline \multicolumn{4}{|l|}{$\mathrm{APH}$} \\
\hline $\begin{array}{l}\text { Yes } \\
\text { No }\end{array}$ & $\begin{array}{l}25 \\
(27.2)\end{array}$ & $\begin{array}{l}7(3.8) \\
177(96.2)\end{array}$ & $32(11.6)$ \\
\hline \multicolumn{4}{|l|}{$\mathrm{PIH}$} \\
\hline $\begin{array}{l}\text { Yes } \\
\text { No }\end{array}$ & $\begin{array}{l}19 \\
(20.7)\end{array}$ & $\begin{array}{l}7(3.8) \\
177(96.8)\end{array}$ & $\begin{array}{l}26(9.4) \\
250(90.6)\end{array}$ \\
\hline \multicolumn{4}{|l|}{ Anemia } \\
\hline $\begin{array}{l}\text { Yes } \\
\text { No }\end{array}$ & $\begin{array}{l}21 \\
(22.8)\end{array}$ & $\begin{array}{l}6(3.3) \\
178(96.7)\end{array}$ & $\begin{array}{l}27(9.8) \\
249(90.2)\end{array}$ \\
\hline Chronic disease & & & \\
\hline
\end{tabular}




\begin{tabular}{|llll|}
\hline Variable & $\begin{array}{l}\text { Cases } \\
(\%)\end{array}$ & $\begin{array}{l}\text { Controls } \\
(\%)\end{array}$ & Total (\%) \\
\hline Yes & 13 & $14(7.6)$ & $27(9.8)$ \\
No & $(14.1)$ & $170(92.4)$ & $249(90.2)$ \\
& 79 & & \\
\hline
\end{tabular}

\section{Intra partum related characteristics of the study participants}

About $43.4 \%$ of the cases and $23.9 \%$ of the controls mother labor were attended by midwives. Majority $54.3 \%$ of the cases and $84.8 \%$ of the controls mother had spontaneous type labor and $50.0 \%$ of cases and $7.6 \%$ of controls mother delivered with stained amniotic fluid. Majority $73.9 \%$ of cases and $88.0 \%$ of controls were cephalic fetal presentation. About $14.1 \%$ of the cases and only $1.1 \%$ of controls were delivered with cored prolapsed (Table 3). 
Table 3

Intra partum related characteristics of the study participants in Debre Berhan referral hospital, Debre Berhan, Ethiopia, 2020

\begin{tabular}{|c|c|c|c|}
\hline Variable & Cases (\%) & Controls (\%) & Total (\%) \\
\hline \multicolumn{4}{|l|}{ Labor attendant } \\
\hline Midwives & $39(43.4)$ & $44(23.9)$ & $83(30.1)$ \\
\hline General practitioner & $30(36.6)$ & $92(50.0)$ & $122(44.2)$ \\
\hline Gynecologist & $23(25.0)$ & $48(26.1)$ & $71(25.7)$ \\
\hline \multicolumn{4}{|l|}{ Type of labor } \\
\hline Spontaneous & $50(54.3)$ & $156(84.8)$ & $206(74.6)$ \\
\hline Induced & $42(45.7)$ & $28(15.2)$ & $70(25.4)$ \\
\hline \multicolumn{4}{|l|}{ Duration of labor } \\
\hline Normal & $24(20.1)$ & $151(82.1)$ & $175(63.4)$ \\
\hline Prolonged & $68(79.9)$ & $33(17.9)$ & $101(36.6)$ \\
\hline \multicolumn{4}{|l|}{ Mode of delivery } \\
\hline SVD & $37(40.2)$ & 131 (71.2) & $168(60.9)$ \\
\hline Instrumental & $26(28.3)$ & $23(12.5)$ & $49(17.7)$ \\
\hline CS & $29(31.5)$ & $30(16.3)$ & $59(21.4)$ \\
\hline \multicolumn{4}{|l|}{ Amniotic fluid } \\
\hline Stained & $46(50.0)$ & $14(7.6)$ & $60(21.7)$ \\
\hline Non stained & $46(50.0)$ & $170(92.4)$ & $216(78.3)$ \\
\hline \multicolumn{4}{|l|}{ PROM } \\
\hline Yes & $39(42.4)$ & $25(13.6)$ & $64(23.2)$ \\
\hline No & $53(57.6)$ & 159 (86.4) & $212(76.8)$ \\
\hline \multicolumn{4}{|l|}{ Obstructed labor } \\
\hline Yes & 27 (29.3) & $16(8.7)$ & $43(15.6)$ \\
\hline No & $65(70.7)$ & 168 (91.3) & $233(84.4)$ \\
\hline \multicolumn{4}{|l|}{ Fetal presentation } \\
\hline Cephalic & $68(73.9)$ & $162(88.0)$ & $230(83.3)$ \\
\hline Not cephalic & $24(26.1)$ & $22(12.0)$ & $46(16.7)$ \\
\hline
\end{tabular}




\begin{tabular}{|llll|} 
Yes & $13(14.1)$ & $2(1.1)$ & $15(5.4)$ \\
No & $79(85.9)$ & $182(98.9)$ & $261(94.6)$ \\
\hline
\end{tabular}

\section{Newborn related characteristics of the study participants}

This study revealed that $54(58.7 \%)$ of cases and $107(58.2 \%)$ of controls were male neonates. Twenty two $(23.9 \%)$ of cases and only $6(3.3 \%)$ of controls were preterm neonates. It was also observed that 39 $(42.4 \%)$ of the cases and $34(18.5 \%)$ of the controls were low birth weight (Table 4).

Table 4

Neonatal related characteristics of the study participants in Debre Berhan referral hospital, Debre Berhan, Ethiopia, 2020

\begin{tabular}{|llll|}
\hline Variable & Cases (\%) & $\begin{array}{l}\text { Controls } \\
(\%)\end{array}$ & Total (\%) \\
\hline $\begin{array}{l}\text { Sex of new } \\
\text { born }\end{array}$ & & & \\
\hline Male & $54(58.7)$ & $107(58.2)$ & $\begin{array}{l}161 \\
(58.3)\end{array}$ \\
Female & $38(41.3)$ & $77(41.8)$ & $\begin{array}{l}115 \\
(41.7)\end{array}$ \\
& & & \\
Gestational & & & $28(10.1)$ \\
age & & & 204 \\
Pre-term & $22(23.9)$ & $6(3.3)$ & $(73.9)$ \\
Term & $38(41.3)$ & $166(90.2)$ & $44(16.0)$ \\
Post-term & $32(34.8)$ & $12(5.5)$ & \\
\hline Birth weight & & & $73(26.4)$ \\
\hline$<2500 g m$ & $39(42.4)$ & $34(18.5)$ & 203 \\
>=2500 & $53(57.6)$ & $150(81.5)$ & $(73.6)$ \\
\hline
\end{tabular}

\section{Determinants of birth asphyxia among newborns}

In bivariate analysis eleven variables showed significantly associated with birth asphyxia. After controlling the possible confounders in multivariate logistic regression analysis maternal education, ANC follow up, presence APH, prolonged labor, stained amniotic fluid, not cephalic fetal presentation and gestational age less than 37 weeks were identified as determinants of birth asphyxia among newborns. 
This study revealed that mother who can't read and write $[A O R=4.7 ; 95 \% \mathrm{Cl}(1.2,11.9)]$ were 4.7 times higher to develop birth asphyxia compared from mother who have college diploma and above. The odds of developing birth asphyxia among mothers who faced antepartum hemorrhage was 7.7 times higher compared from the counterparts $[\mathrm{AOR}=7.7 ; 95 \% \mathrm{Cl}(1.5,18.5)]$. This study revealed that prolonged labor was the main predictor of birth asphyxia. A mother who had prolonged labor was more than 13 time higher risk compared from normal labor on the outcome of birth asphyxia [AOR $=13.5 ; 95 \% \mathrm{Cl}(2.0,19.4)]$. Preterm babies were 4.1 times higher risk than term babies to development birth asphyxia [AOR $=4.1$; $95 \% \mathrm{Cl}(1.8,9.2)]$ (Table 5). 
Table 5

Bivariate and Multivariate logistic regression analysis on the determinants of birth asphyxia among newborns in Debre Berhan Referral hospital, Debre Berhan, Ethiopia 2020

\begin{tabular}{|c|c|c|c|c|}
\hline \multirow[b]{2}{*}{ Variable } & \multicolumn{2}{|c|}{ Asphyxia status } & \multirow[b]{2}{*}{ COR $(95 \% \mathrm{Cl})$} & \multirow[b]{2}{*}{ AOR $(95 \% \mathrm{Cl})$} \\
\hline & Cases (\%) & Controls (\%) & & \\
\hline \multicolumn{5}{|l|}{ Maternal education } \\
\hline Can't read \& write & $39(42.4)$ & $11(6.0)$ & $14.8(6.4,34.4)$ & $4.7(1.2,11.9)^{\star}$ \\
\hline Primary school & $22(23.9)$ & $27(14.6)$ & $3.4(1.6,7.3)$ & $0.5(0.1,2.2)$ \\
\hline Secondary school & $13(14.1)$ & $71(38.6)$ & $0.8(0.4,1.7)$ & $0.5(0.4,2.4)$ \\
\hline Diploma \& above & 18 (19.6) & $75(40.8)$ & 1.0 & 1.0 \\
\hline \multicolumn{5}{|l|}{ ANC follow up } \\
\hline$\leq$ Two & $39(42.4)$ & $24(13.1)$ & $6.9(3.7,13.1)$ & $4.6(1.1,9.5)^{\star}$ \\
\hline Three & $20(21.7)$ & $19(10.3)$ & $4.5(2.2,9.4)$ & $2.4(0.5,11.4)$ \\
\hline Four and above & $33(35.9)$ & $141(76.6)$ & 1.0 & 1.0 \\
\hline \multicolumn{5}{|l|}{ APH } \\
\hline Yes & $25(27.2)$ & $7(3.8)$ & $9.4(3.9,22.8)$ & $7.7(1.5,18.5)^{\star}$ \\
\hline No & $67(72.8)$ & $177(96.2)$ & 1.0 & 1.0 \\
\hline \multicolumn{5}{|l|}{ Type of labor } \\
\hline Spontaneous & $50(54.3)$ & $156(84.8)$ & 1.0 & 1.0 \\
\hline Induced & $42(45.7)$ & $28(15.2)$ & $4.7(1.1,7.4)$ & $6.4(0.7,37.2)$ \\
\hline \multicolumn{5}{|l|}{ Duration of labor } \\
\hline Normal & $24(26.1)$ & $151(82.1)$ & 1.0 & 1.0 \\
\hline Prolonged & 68 (73.9) & $33(13.9)$ & $13.0(2.4,18.1)$ & $13.5(2.0,19.4)^{\star}$ \\
\hline \multicolumn{5}{|l|}{ Amniotic fluid } \\
\hline Stained & $46(50.0)$ & $14(7.6)$ & $12.1(6.2,24.0)$ & $11.3(2.7,39.5)^{\star}$ \\
\hline Non stained & $46(50.0)$ & $170(92.4)$ & 1.0 & 1.0 \\
\hline \multicolumn{5}{|l|}{ PROM } \\
\hline Yes & $39(42.4)$ & $25(13.6)$ & $4.7(2.6,8.5)$ & $6.1(0.7,8.5)$ \\
\hline No & $53(57.6)$ & $159(86.4)$ & 1.0 & 1.0 \\
\hline \multicolumn{5}{|l|}{ Obstructed labor } \\
\hline Yes & 27 (29.3) & $16(8.7)$ & $4.4(2.2,8.6)$ & $1.2(0.1,2.0)$ \\
\hline
\end{tabular}




\begin{tabular}{|lllll|} 
No & $65(70.7)$ & $168(91.3)$ & 1.0 & 1.0 \\
Fetal presentation & & & & 1.0 \\
Cephalic presentation & $68(73.9)$ & $162(88.0)$ & 1.0 & $4.5(2.0,8.4)^{\star}$ \\
\hline Breech presentation & $24(26.1)$ & $22(12.0)$ & $2.6(2.0,7.3)$ & \\
Gestational age & & & & $4.1(1.8,9.2)^{\star}$ \\
Pre-term (<37) & $22(23.9)$ & $6(3.3)$ & $1.4(1.2,6.1)$ & $0.1(0.01,0.5)$ \\
Perm (37-39) & $38(41.3)$ & $166(90.2)$ & $0.2(0.1,1.3)$ & 1.0 \\
Birth weight & $32(34.8)$ & $12(6.5)$ & 1.0 & $2.7(0.9,8.5)$ \\
$<2500$ gm & & & & 1.0 \\
$>=2500$ & $39(42.4)$ & $34(18.5)$ & $3.3(1.9,5.7)$ & 1.0 \\
\hline
\end{tabular}

\section{Discussion}

This study tried to identify the determinants of birth asphyxia among newborns in Debre Berhan referral hospital by including number of variables from different categories like socio-demographic, antepartum, intra partum and neonatal related characteristics of the newborns.

This study revealed that mothers who can't read and write were 4.7 times higher risk to have asphyxiated newborns compared to mothers who have a college diploma and above. This finding was consistent with the study conducted in Pakistan [16] and Ethiopia [7, 12, 17]. This could indicate that poor literacy limits mothers from having independent decisions and good access to family resources that could restrict their health-seeking behavior during the antepartum period and also it is an indicator of poor socio-economic status of the community and unplanned pregnancy.

Gestational age was significantly associated with birth asphyxia. Preterm ( $<37 \mathrm{wks})$ was 4.1 times more likely asphyxiated than the term newborns. This finding was consistent with those of previous studies conducted in Pakistan [16], Nepal [18], and Ethiopia [9, 12, 19]. This may be due to the fact that preterm babies face multiple morbidities including organ system, immaturity especially lung immaturities causing respiratory failure.

Newborns with the breech presentation were 4.5 times the odds of developing birth asphyxia than the cephalic presentation. This finding was consistent with previous studies conducted in Uganda [20], Cameron [21], and Ethiopia [22]. Mal-presentation of foetus is associated with premature rupture of membrane; this premature rupture of membrane could leads to umbilical cord accidents occur with sub sequent asphyxia at birth [14]. But, the finding was inconsistent with previous studies conducted in Ethiopia $[13,23]$. This difference might be due to the difference in the study population and the study design. 
Prolonged labour was significantly associated with birth asphyxia. This finding was consistent with studies conducted in Cameron [21], Pakistan [16], and Ethiopia [11, 24-26]. This could be due to primary, secondary, or tertiary dalliance. This is in fact that, when labour is prolonged there was a high probability for the foetus to become distressed which can lead to birth asphyxia [26].

Less than or equals to two ANC follow up by the mothers were 4.6 times higher odds of developing asphyxiated newborns than mothers who have more than four ANC follow up. This finding was inconsistent with the study conducted in Ethiopia [13,23,25]. This difference may be due to the number of health facilities included in the study and study period. This ANC visit of the pregnant mother are very important to minimize adverse pregnancy outcomes including birth asphyxia as they provide chance for evaluating the foetal wellbeing and permit management soon by improving the health and wellbeing of the mother and preventing further complications by early detection and treatment of diseases.

In this study, we have limitations that should be noted. The study was done in single health facility therefore; it is difficult to generalize for the whole country with this small sample. This study also subjected to recalling bias of mothers when they remembered their previous history.

\section{Conclusions}

In this study, maternal education can't read \& write, antepartum hemorrhage, prolonged labour, stained amniotic fluid, breech fetal presentation, preterm birth were significantly associated with birth asphyxia. So, educating mothers to have continuous follow-up during the pregnancy period and provide quality care to the women in labour through close monitoring of the fetus presentation were recommended to reduce birth asphyxia.

\section{Abbreviations}

ANC - Antenatal Care, AOR- Adjusted Odd Ratio, DDS - Dietary Diversity Score, FAO- Food and Agriculture Organization, NNP- National Nutritional Program, SD - Standard Deviation, SPSS - Statistical Package for Social Science, WHO - World Health Organization

\section{Declarations}

\section{Ethical approval and consent to participate}

This study was carried out after getting ethical clearance from Debre Berhan University, Institute of medicine and Health Science, College of Health Science, Department of Public Health research ethics review committee Ref. No./IOMHS/029/201/10/2020. Data collection was carried out after receiving ethical clearance letter from the district administrative health bureau. Informed written consent was obtained from each study participant prior to data collection. Each participant read the letter and explained further by the data collectors for easily understands the objective of the research. Finally, the consent form was signed by each participant. 


\section{Consent to publication}

Not applicable.

\section{Availability of data and material}

The datasets used and/or analyzed during the current study available from the corresponding author on reasonable request.

\section{Competing interests}

The Authors declare that we do not have any financial or non-financial competing interests in reference to this article for its publication.

\section{Funding}

Not applicable

\section{Authors' contributions}

SST supervised the design of the study, writing and critically reviewed the drafts. YTG conceptualized and designed the study, obtain fund for the study, coordinate, and monitor the overall implementation of the project. She also participated in data extraction and analysis, interpreted the results and wrote the initial draft. SMA and AST contributed to the design and write of subsequent drafts of the study. All authors read and approved the final manuscript.

\section{Acknowledgements}

We would like to thank Debre Berhan Referral hospital medical directors for their collaboration during conducting this research. Our gratitude also goes to our data collectors and study participants for their willingness to participate in the study. At last but not least, we would like to forward our special thank and sincere appreciations to our friends who gave advice and comment throughout the whole research project activities.

\section{References}

1. Spector JM, Daga S: Preventing those so-called stillbirths. Bulletin of the World Health Organization 2008, 86:315-316.

2. Solnes Miltenburg A: Quality of Maternity Care in Rural Tanzania: Understanding Local Realities and Identification of Opportunities for Improvement. 2019.

3. Organization WH: Newborn death and illness. Millennium Development goal (mdg) 2011, 4.

4. Fekede A: Knowledge, Attitude and Practice Towards Management of Birth Asphyxia among Nurses and Midwives Working In Labour Ward and Nicu in Governmental Hospitals, Addis Ababa, Ethiopia, 
2017. Addis Ababa University; 2017.

5. EDHS: Demographic and health survey key indicators 2016.

6. Kiyani AN, Arshad Khushdil AE: Perinatal factors leading to birth asphyxia among term newborns in a tertiary care hospital. Iranian journal of pediatrics 2014, 24(5):637.

7. Wosenu L, Worku AG, Teshome DF, Gelagay AA: Determinants of birth asphyxia among live birth newborns in University of Gondar referral hospital, northwest Ethiopia: A case-control study. PloS one 2018, 13(9):e0203763.

8. MoH F: National strategy for child survival in Ethiopia. Addis Ababa, Ethiopia 2005.

9. Abdo RA, Halil HM, Kebede BA, Anshebo AA, Gejo NG: Prevalence and contributing factors of birth asphyxia among the neonates delivered at Nigist Eleni Mohammed memorial teaching hospital, Southern Ethiopia: a cross-sectional study. BMC pregnancy and childbirth 2019, 19(1):1-7.

10. Alemu A, Melaku G, Abera GB, Damte A: Prevalence and associated factors of perinatal asphyxia among newborns in Dilla University referral hospital, Southern Ethiopia-2017. Pediatric Health, Medicine and Therapeutics 2019, 10:69.

11. Gebregziabher GT, Hadgu FB, Abebe HT: Prevalence and associated factors of perinatal asphyxia in neonates admitted to ayder comprehensive specialized hospital, Northern Ethiopia: a cross-sectional study. International journal of pediatrics 2020, 2020.

12. Tasew H, Zemicheal M, Teklay G, Mariye T, Ayele E: Risk factors of birth asphyxia among newborns in public hospitals of Central Zone, Tigray, Ethiopia 2018. BMC research notes 2018, 11(1):1-7.

13. Mulugeta T, Sebsibe G, Fenta FA, Sibhat M: Risk factors of perinatal asphyxia among newborns delivered at public hospitals in Addis Ababa, Ethiopia: case-control study. Pediatric Health, Medicine and Therapeutics 2020, 11:297.

14. Bayih WA, Yitbarek GY, Aynalem YA, Abate BB, Tesfaw A, Ayalew MY, Belay DM, Hailemeskel HS, Alemu AY: Prevalence and associated factors of birth asphyxia among live births at Debre Tabor General Hospital, North Central Ethiopia. BMC pregnancy and childbirth 2020, 20(1):1-12.

15. ABABA A: Federal Democratic Republic of Ethiopia Ministry of Health CLTSH Verification and Certification Protocol. Federal Democratic Republic of Ethiopia Ministry of Health CLTSH Verification and Certification Protocol 2012.

16. Tabassum F, Rizvi A, Ariff S, Soofi S, Bhutta ZA: Risk factors associated with birth asphyxia in rural district Matiari, Pakistan: a case control study. International Journal of Clinical Medicine 2014, 5(21):1430.

17. Desalew A, Semahgn A, Tesfaye G: Determinants of birth asphyxia among newborns in Ethiopia: A systematic review and meta-analysis. International journal of health sciences 2020, 14(1):35.

18. Lee AC, Mullany LC, Tielsch JM, Katz J, Khatry SK, LeClerq SC, Adhikari RK, Shrestha SR, Darmstadt GL: Risk factors for neonatal mortality due to birth asphyxia in southern Nepal: a prospective, community-based cohort study. Pediatrics 2008, 121(5):e1381-e1390. 
19. Yeshaneh A, Kassa A, Kassa ZY, Adane D, Fikadu Y, Wassie ST, Alemu BW, Tadese M, Shitu S, Abebe $\mathrm{H}$ : The determinants of 5 th minute low Apgar score among newborns who delivered at public hospitals in Hawassa City, South Ethiopia. BMC pediatrics 2021, 21(1):1-10.

20. Kaye D: Antenatal and intrapartum risk factors for birth asphyxia among emergency obstetric referrals in Mulago Hospital, Kampala, Uganda. East african medical journal 2003, 80(3):140-143.

21. Chiabi A, Nguefack S, Evelyne M, Nodem S, Mbuagbaw L, Mbonda E, Tchokoteu P-F: Risk factors for birth asphyxia in an urban health facility in Cameroon. Iranian journal of child neurology 2013, 7(3):46.

22. Kune G, Oljira H, Wakgari N, Zerihun E, Aboma M: Determinants of birth asphyxia among newborns delivered in public hospitals of West Shoa Zone, Central Ethiopia: A case-control study. PloS one 2021, 16(3): e0248504.

23. Woday A, Muluneh A, St Denis C: Birth asphyxia and its associated factors among newborns in public hospital, northeast Amhara, Ethiopia. PloS one 2019, 14(12):e0226891.

24. Kibret Y, Hailu G, Angaw K: Determinants of Birth-Asphyxia among Newborns in Dessie Town Hospitals, North-Central Ethiopia, 2018. 2017.

25. Meshesha AD, Azage M, Worku E, Bogale GG: Determinants of Birth asphyxia among newborns in referral hospitals of Amhara National Regional State, Ethiopia. Pediatric Health, Medicine and Therapeutics 2020, 11:1.

26. Sendeku FW, Azeze GG, Fenta SL: Perinatal asphyxia and its associated factors in Ethiopia: a systematic review and meta-analysis. BMC pediatrics 2020, 20(1):1-11. 